\title{
Can the NHSN dialysis event protocol be implemented in an Irish dialysis unit?
}

\author{
Raazi Bajwa' ${ }^{1}$ Anne Casey², Cathail Collier², Patrick O'Kelly², Peter Conlon², Fidelma Fitzpatrick ${ }^{3}$ \\ ${ }^{1}$ Royal College of Surgeons in Ireland, Dublin, Ireland \\ ${ }^{2}$ Department of Nephrology, Beaumont Hospital, Ireland \\ ${ }^{3}$ Department of Microbiology, Beaumont Hospital. Ireland and Health Protection Surveillance Centre, \\ Dublin, Ireland
}

\begin{abstract}
Infection is the second most common cause of death in patients with chronic kidney disease and so International guidelines recommend surveillance of infection in dialysis units. This study examines the feasibility of establishing and maintaining the National Healthcare Safety Network (NSHN) dialysis event protocol in our hospital.
\end{abstract}

Two outpatient haemodialysis wards were surveyed for six weeks using the NHSN dialysis events protocol. Numerator data were collected by recording dialysis events; hospitalisation, intravenous (IV) antimicrobial start or a positive blood culture.

Hospitalisations, IV antimicrobial starts and positive blood cultures were recorded at rates of 13, 8.52 and 3.14 per 100 patient months, respectively. Seven vascular access infections; six access associated bacteraemias and one complication (infective endocarditis) were recorded. Dialysis events were more common in patient with IV catheters when compared with patients with fistulas ( $p<0.001)$. The rate of IV antimicrobial starts was significantly higher than NHSN rates $(p=0.001)$. NHSN surveillance protocol use allows the establishment of standardised baseline dialysis event rates and has highlighted antimicrobial use and higher event rates in patients with IV catheters as areas for improvement. On-going surveillance is feasible, though dependent on multidisciplinary dialysis staff involvement and ownership and the presence of a surveillance coordinator.

\section{Key words}

Hemodialysis unit, hospital; Infection control, standards; cross infection, prevention and control; safety management, methods

\section{Corresponding Author}

Raazi Bajwa

Royal College of Surgeons in Ireland, Dublin, Ireland

Email: raazibajwa@rcsi.ie 


\section{Introduction}

Infection is second only to cardiovascular disease as the most common cause of death in patients with chronic kidney disease. ${ }^{1}$ Significant proportions of these patients receive haemodialysis via an intravascular catheter and are therefore at risk of catheter-related bloodstream infection. Bloodstream infection (BSI) in haemodialysis patients can be life threatening and associated with substantial morbidity and costs. ${ }^{2}$ Antimicrobial stewardship in dialysis units is essential as inappropriate antimicrobial use contributes to the emergence of multidrug resistant organisms, such as meticillin resistant Staphylococcus aureus (MRSA), a frequent cause of BSI. In our hospital, S. aureus BSI are monitored quarterly; however, we do not have a standardised internationally comparable haemodialysis infection surveillance system such as that in other countries. ${ }^{3}$ Standardised surveillance of infection and antimicrobial usage in this patient population is an essential component of a healthcareassociated infection (HCAl) prevention programme as it establishes baseline rates and helps dialysis units track the effect of improvement programmes. ${ }^{4,5}$

The National Healthcare Safety Network (NHSN) in the US coordinates dialysis surveillance via the dialysis event protocol and reported increased BSI rates in patients with permanent and temporary catheters (3.1 and 17.8 per 100 patient months respectively) in comparison to patients with fistulas or grafts ( 0.5 and 0.9 per 100 patient months). ${ }^{6}$ Other countries have also shown an association of BSI in dialysis patients with catheter use. ${ }^{7}$ Embedding a dialysis specific surveillance scheme in a unit is associated with reductions in BSI and antimicrobial consumption and also improves staff awareness of infection prevention and control and the importance of optimising vascular access. $^{7}$

The aim of our study was to examine the feasibility of establishing and maintaining the NHSN dialysis event protocol in our hospital, with a view to establishing baseline rates, highlighting areas for improvement and evaluating the effectiveness of our HCAI prevention and antimicrobial stewardship programmes.

\section{Methods}

Our hospital is an 820-bedded tertiary referral centre containing the national referral centre for neurosurgical patients, renal and pancreatic transplantation, cochlear implantation and a regional treatment centre for ear, nose and throat and gastroenterology patients. There are two outpatient haemodialysis wards and one inpatient haemodialysis ward, consisting of 20 and four beds, respectively. The number of patients receiving outpatient haemodialysis has been steadily rising with a total number of 197 in 2010. In contrast, the average number of outpatients per centre providing data to the NHSN is $73,{ }^{6}$ while 112 outpatients were recorded as receiving dialysis at Hammersmith Hospital, UK in $2002 .^{7}$

Prospective surveillance using the NHSN dialysis event protocol was conducted in two of three chronic haemodialysis wards for six weeks from 15th March to 23rd April, 2010. ${ }^{3}$ Numerator and denominator data were collected on two specifically designed paper forms (dialysis event and denominator forms) and inputted into a Microsoft Excel database. A month was defined as 28 days. For the first four weeks, patient-months were calculated on the first two days of surveillance by counting the number of patients receiving haemodialysis and also recording their haemodialysis access route. Likewise, for the last two weeks, similar was done, but all values halved. These figures were then added to the first four week's figures to get the total patient-months for the duration of the surveillance.

Numerator data was collected in the form of dialysis events. A dialysis event was defined as any patient who required hospitalisation, had an intravenous (IV) antimicrobial start or a positive blood culture. Hospitalisations included all hospitalisations that involved an overnight stay. Each time a patient was hospitalised, no matter how soon after the last hospitalisation it was entered as a new event. If the patient was hospitalised and returned to the dialysis unit on IV antimicrobials, both were included in the same event. Positive blood cultures (causative organism and antimicrobial susceptibility) drawn as 
an outpatient or within one day of hospital admission were recorded. Local access infection, access associated bacteraemia and vascular access infections were defined as outlined in the NHSN protocol. ${ }^{3}$

Multidisciplinary dialysis staff education sessions were conducted before the study commenced outlining study aims and the NHSN dialysis event protocol. Dialysis staff was requested to notify the surveillant (RB) if patients were considered to have a dialysis event. In order to ensure all events were documented, a daily census of all renal admissions and a daily list of all positive blood cultures from dialysis patients were obtained. Upon a dialysis event being recognised, a dialysis event form was completed. Patient follow up was performed by medical and nursing chart review, daily discussions with dialysis staff and daily review of the dialysis unit information system database. Dialysis event rates were calculated as described in the NHSN protocol and expressed per 100 patientmonths. Rates were expressed as crude rates and after standardisation for access type and compared with comparable published rates. Rates were calculated as per 100 patient-months. Rate ratios were calculated by dividing the rate in question by the comparable rate of either the NHSN or Hammersmith Hospital, UK.

\section{Results}

In total, 222.5 patient-months (113.5 fistula, 104.5 permanent catheter and 4.5 graft patient-months) were recorded over the study period. No patients received dialysis via a temporary catheter. Dialysis events documented were hospitalisations (29), IV antimicrobial starts (19) and positive blood cultures (seven). Of the seven positive blood cultures (all drawn from an IV catheter); four grew meticillin susceptible $S$. aureus and three coagulase negative staphylococci. Of note, three also had positive IV tip cultures (two $S$. aureus and one vancomycin-resistant enterococci). In addition, there were seven vascular access infections; one local access infection and six access-associated bacteraemias. Seven catheter removals were recorded and one patient had infective endocarditis diagnosed by transoesophageal echocardiography, which was recorded as a complication.

Analyses of dialysis event rates involved comparisons to published NHSN, and Hammersmith Hospital,
UK data by means of incidence rate ratios. ${ }^{6,7}$ Table I compares arteriovenous fistula (AVF) dialysis events to IV catheters' in our unit. There were significantly more dialysis events in patients with IV catheters than those with an AVF. Table II outlines the distribution of dialysis event rates by vascular access category and comparisons with NHSN and Hammersmith Hospital, UK rates. ${ }^{7}$ Similar dialysis event rates were recorded apart from higher IV antimicrobial starts in our hospital when compared to the corresponding NHSN rate..$^{6,7}$

\section{Discussion}

The use of the NHSN surveillance protocol allowed the establishment of standardised baseline dialysis event rates in our haemodialysis unit. Initial results can be accurately compared to future months' rates and to other units that employ the same protocol. The high rate of empiric IV antimicrobial use and the rate of dialysis events in patients with IV catheters have been highlighted and represent areas where improvements can be made in order to optimise patient quality and safety.

The rate of dialysis events was significantly higher in patients with IV catheters when compared with patients with fistulas (Table II). While the choice of dialysis access depends on many factors, a primary AVF if it can be created is usually the most preferable access and is recommended by national and international guidelines. ${ }^{4,8}$ It provides the most durable long term access with the least complications or interventions required to maintain patency. ${ }^{6,7,9}$ Such strategies of preemptive management of vascular access results in long term survival advantages for patients and reductions in BSI rates. However, dialysis units require adequate access to a vascular surgical service in order to ensure the timely creation of primary vascular access. Where AVF access isn't possible, aseptic technique and hand hygiene is essential during catheter insertion and care. As a result of our pilot study we have intensified our efforts at promoting AVF among our patients and introduced a number of IV catheter improvement programmes including catheter insertion checklists and care bundles. The IV antimicrobial start rate was more than double the NHSN published rates and when considered in the context of NHSN percentiles, our hospital would represent a high outlier (>90th)..$^{10}$ While crude comparisons of Irish with NHSN rates 


\section{Table I. Intravenous (IV) catheter dialysis event rates in comparison to arteriovenous fistula dialysis event rates}

\begin{tabular}{|c|c|c|c|}
\hline Event & Patient-months & Dialysis event rate ${ }^{a}$ & $\begin{array}{l}\text { Incidence rate ratio } \\
(95 \% \mathrm{Cl})\end{array}$ \\
\hline \multicolumn{4}{|l|}{ Hospitalisation } \\
\hline IV catheter & 113.5 & 23.81 & $6.72(2.32-26.59)$ \\
\hline Fistula & 104.5 & 3.53 & $p<0.001$ \\
\hline \multicolumn{4}{|c|}{ IV antimicrobial starts } \\
\hline IV catheter & 113.5 & 16.19 & $9.15(2.17-81.63)$ \\
\hline Fistula & 104.5 & 1.77 & $p=0.002$ \\
\hline \multicolumn{4}{|c|}{ Positive blood culture } \\
\hline IV catheter & 113.5 & 6.67 & $\infty$ \\
\hline Fistula & 104.5 & 0 & $p=0.006$ \\
\hline \multicolumn{4}{|l|}{ All events } \\
\hline IV catheter & 113.5 & 46.67 & $8.79(3.76-25.11)$ \\
\hline Fistula & 104.5 & 5.3 & $p<0.001$ \\
\hline
\end{tabular}

are difficult because of potential differences in patient case mix, dialysis care provision and practice, it does represent an area that warrants future investigation. For example, our study did not evaluate the appropriateness of empiric IV antimicrobials or the blood culture contamination rate, however, by highlighting these areas, further analysis of antimicrobial prescribing practices and staff education regarding antimicrobial stewardship principles and aseptic techniques will ultimately improve patient care and quality. Appropriate antimicrobial prescribing is essential in order to limit the emergence of antimicrobial resistant organisms such as MRSA in this patient population. ${ }^{11}$ Our pilot surveillance scheme increased awareness of IV-related infection and the principles of infection prevention in our unit. Feedback of results facilitated several improvement programmes relating to IV care such as mandatory IV insertion checklists. However, the success of the pilot was dependent on embedding the programme within daily practice in the dialysis unit. In order to continue the surveillance successfully, a broad based approach and multidisciplinary co-ordination is necessary. Essential components of our programme included the presence of a lead surveillance coordinator, timely feedback of results and involvement of medical and nursing dialysis staff in collection of surveillance data and planning improvement programmes. Establishing an on-going standardised surveillance programme in our haemodialysis unit will be an important step in improving patient safety and quality of care. Lessons learnt from our pilot will be key to embedding the surveillance programme in the unit's activities. In the present resource constrained environment, it will be difficult to attract additional resources' to continue the programme, specifically the appointment of dedicated sessions for a surveillance coordinator. We are currently evaluating how we can embed the scheme in the day to day activities of the unit by distributing key surveillance tasks to dialysis staff and linking with our infection prevention and control service. We plan to continue the surveillance scheme on intermittent periodic basis in the meantime, in order to track the effect of our preventative programmes.

The strengths of the study included a full time data collector ensuring consistency in collection, interpretation of data and a number of methods in place which ensured that dialysis events weren't missed. Initial multidisciplinary staff education sessions coupled with on-going liaison for the course of the surveillance fostered local ownership and 


\begin{tabular}{|c|c|c|c|c|c|}
\hline & $\begin{array}{l}\text { Beaumont } \\
\text { Hospital }\end{array}$ & $\mathrm{NHSN}^{6}$ & $\begin{array}{l}\text { Incidence rate ratio } \\
(95 \% \mathrm{Cl})\end{array}$ & $\begin{array}{l}\text { Hammersmith } \\
\text { Hospital }^{7}\end{array}$ & $\begin{array}{l}\text { Incidence rate ratio } \\
(95 \% \mathrm{Cl})\end{array}$ \\
\hline Hospitalisation & 13 & 10.64 & $\begin{array}{l}1.22(0.82-1.76) \\
p=0.286\end{array}$ & 14.89 & $\begin{array}{l}0.87(0.54-1.38) \\
p=0.557\end{array}$ \\
\hline IV catheter & 23.81 & 15.69 & $\begin{array}{l}1.52(0.98-2.25) \\
p=0.05\end{array}$ & & \\
\hline Fistula & 3.54 & 7.69 & $\begin{array}{l}0.46(0.12-1.18) \\
p=0.093\end{array}$ & & \\
\hline $\begin{array}{l}\text { IV antimicrobial } \\
\text { starts }\end{array}$ & 8.52 & 3.48 & $\begin{array}{l}2.45(1.47-3.84) \\
p<0.001\end{array}$ & 7.69 & $\begin{array}{l}0.99(0.52-1.85) \\
p=0.988\end{array}$ \\
\hline IV catheter & 16.19 & 6.44 & $\begin{array}{l}2.51(1.45-4.06) \\
p=0.001\end{array}$ & & \\
\hline Fistula & 1.77 & 1.8 & $\begin{array}{l}0.98(0.12-3.59) \\
p=1\end{array}$ & & \\
\hline $\begin{array}{l}\text { Positive blood } \\
\text { cultures }\end{array}$ & 3.14 & 1.89 & $\begin{array}{l}1.65(0.66-3.43) \\
p=0.20\end{array}$ & $6.2^{*}$ & $\begin{array}{l}0.51(0.18-1.20) \\
p=0.102\end{array}$ \\
\hline IV catheter & 6.67 & 4.25 & $\begin{array}{l}1.57(0.63-3.26) \\
p=0.253\end{array}$ & & \\
\hline Fistula & 0 & 0.52 & $\begin{array}{l}0 \\
p=0.557\end{array}$ & & \\
\hline
\end{tabular}

*Only access-related bacteraemia

IV: Intravenous

clinical engagement. Limitations of the study are its short surveillance period and that all hospitalisations are recorded, despite the fact that they may be completely unrelated to the patient's renal disease or haemodialysis vascular access. However, we decided from the outset to follow the NHSN protocol to enable true comparisons, hence why we recorded all hospitalisations.

Our pilot study has demonstrated that implementation of a standardised dialysis surveillance scheme is feasible in a busy dialysis unit. However, multidisciplinary dialysis staff involvement and the presence and ownership of a surveillance coordinator are critical for successful implementation and to drive improvement programmes in the future. Results have highlighted the importance of optimising vascular access, appropriate care of IV catheters and the necessity to improve antimicrobial stewardship in the unit. Surveillance is an essential part of infection prevention and control and should be part of quality care for all dialysis units. While there are caveats with international comparisons as discussed above, we have established a baseline that will facilitate us to demonstrate the effect of future infection prevention and control and antimicrobial stewardship strategies.

\section{References}

1. U S Renal Data System. USRDS 2011 Annual Data Report: Atlas of Chronic Kidney Disease and End-Stage Renal Disease in the United States. National Institutes of Health, National Institute of Diabetes and Digestive and Kidney Diseases, Bethesda, Maryland. http://www.usrds.org/reference.aspx [Accessed April 5, 2011]

2. Patel PR. Epidemiology, Surveillance, and Prevention of Bloodstream Infections in Hemodialysis Patients. American Journal of Kidney Diseases 2010; 56(3) 566-577. http:// dx.doi.org/10.1053/j.ajkd.2010.02.352 
3. Centers for Disease Control and Prevention. CDC - Dialysis Event - NHSN, June 2011. Public Health Service, US Department of Health and Human Services, Centers for Disease Control and Prevention, Atlanta, Georgia. http:// www.cdc.gov/nhsn/psc_da_de.html [Accessed July 6, 2011]

4. National Kidney Foundation. Clinical Practice Guidelines and Clinical Practice Recommendations. National Kidney Foundation. http://www.kidney.org/professionals/KDOQl/ guideline_upHD_PD_VA/index.htm [Accessed March 27, 2010]

5. O'Grady NP. Guidelines for the Prevention of Intravascular Catheter-Related Infections. Public Health Service, US Department of Health and Human Services, Centers for Disease Control and Prevention, Atlanta, Georgia. http:// www.cdc.gov/mmwr/preview/mmwrhtml/rr5110a1.htm [Accessed December 8, 2010]

6. Klevens RM. Dialysis surveillance Report: national healthcare safety network (NHSN)—Data summary for 2006. Seminars in Dialysis 2006; 21(1): 24-28. http://dx.doi.org/10.1111/ j.1525-139X.2007.00379.x
7. George A. Reducing dialysis associated bacteraemia, and recommendations for surveillance in the United Kingdom: prospective study. British Medical Journal 2006; 332(7555): 1435 -1439. http://dx.doi.org/10.1136/bmj.332.7555.1435

8. Health Protection Surveillance Centre. Prevention of Intravascular Catheter-related infection in Ireland. Health Protection Surveillance Centre, Dublin, Ireland. http://www. hpsc.ie/hpsc/AZ/MicrobiologyAntimicrobialResistance/ InfectionControlandHAI/IntravascularIVlines/Publications/ File,4115,en.pdf [Accessed March 1, 2010]

9. Stevenson KB. Standardized surveillance of hemodialysis vascular access infections: 18-month experience at an outpatient, multifacility hemodialysis center. Infection Control and Hospital Epidemiology 2000; 21(3): 200-203. http://dx.doi.org/10.1086/501744

10. Edwards JR. National Healthcare Safety Network (NHSN) report: Data summary for 2006 through 2008, issued December 2009. American Journal of Infection Control 2009; 37(10): 783-870. http://dx.doi.org/10.1016/j. ajic.2009.10.001

11. Lee SC. An outbreak of methicillin-resistant Staphylococcus aureus infections related to central venous catheters for haemodialysis. Infection Control and Hospital Epidemiology 2004; 25(8): 678-684. http://dx.doi.org/10.1086/502462 